\title{
NK cells in childhood obesity are activated, metabolically stressed, and functionally deficient
}

\author{
Laura M. Tobin, ${ }^{1,2}$ Meenal Mavinkurve, ${ }^{1,2}$ Eirin Carolan,, ${ }^{1,2}$ David Kinlen, ${ }^{1,2}$ Eoin C. O'Brien, ${ }^{3}$ \\ Mark A. Little, ${ }^{3}$ David K. Finlay, ${ }^{4}$ Declan Cody, ${ }^{2,5}$ Andrew E. Hogan ${ }^{1,2,6}$ and Donal O'Shea \\ 'Obesity Immunology Group, Education and Research Centre, St. Vincent's University Hospital, University College Dublin, \\ Dublin, Ireland. ${ }^{2}$ National Children's Research Centre, Dublin, Ireland. ${ }^{3}$ Trinity Health Kidney Centre and ${ }^{4}$ Trinity Biomedical \\ Sciences Institute, School of Biochemistry and Immunology, Trinity College Dublin, Dublin, Ireland. ${ }^{5}$ Department of \\ Diabetes \& Endocrinology, Our Lady's Children's Hospital Crumlin, Dublin, Ireland. IInstitute of Immunology, Department \\ of Biology, Maynooth University, Maynooth, Co Kildare, Ireland.
}

\begin{abstract}
Childhood obesity is a major global concern, with over $\mathbf{5 0}$ million children now classified as obese. Obesity has been linked to the development of numerous chronic inflammatory diseases, including type 2 diabetes and multiple cancers. NK cells are a subset of innate effector cells, which play an important role in the regulation of adipose tissue and antitumor immunity. NK cells can spontaneously kill transformed cells and coordinate subsequent immune responses through their production of cytokines. We investigated the effect of obesity on NK cells in a cohort of obese children, compared to children with a healthy weight. We demonstrated a reduction in peripheral NK cell frequencies in childhood obesity and inverse correlations with body mass index and insulin resistance. Compared with NK cells from children with normal weight, we show increased NK cell activation and metabolism in obese children (PD-1, mTOR activation, ECAR, and mitochondrial ROS), along with a reduced capacity to respond to stimulus, ultimately leading to loss of function (proliferation and tumor lysis). Collectively we show that NK cells from obese children are activated, metabolically stressed, and losing the ability to perform their basic duties. Paired with the reduction in NK cell frequencies in childhood obesity, this suggests that the negative effect on antitumor immunity is present early in the life course of obesity and certainly many years before the development of overt malignancies.
\end{abstract}

Authorship note: L.M. Tobin and M. Mavinkurve are co-first authors. A.E. Hogan and D. O'Shea are co-senior authors.

Conflict of interest: The authors have declared that no conflict of interest exists.

Submitted: May 9, 2017

Accepted: November 16, 2017

Published: December 21, 2017

Reference information:

JCI Insight. 2017;2(24):e94939.

https://doi.org/10.1172/jci.

insight.94939.

\section{Introduction}

Childhood obesity is increasing in prevalence, and longitudinal studies have shown that elevated BMI tracks strongly from childhood into adulthood, increasing the future risk of chronic disease such as type 2 diabetes mellitus (T2DM), cardiovascular disease, and certain cancers (1). Twenty percent of adult cancer cases are attributed to being overweight or obese (2). In obese adult populations, increased incidences of multiple cancer types are described, including oesophageal, pancreatic, ovarian, renal cell carcinoma, endometrial, and certain hematopoietic malignancies (3). In obese children, there are reports of worse outcomes in hematological cancers, such as acute lymphoblastic leukemia and acute myeloblastic leukemia (4, 5). Additionally, larger body size in childhood and adolescence is associated with increased risk of non-Hodgkin's lymphoma later in life (6). A recent study that investigated cancer rates in a cohort of 1.79 million Israeli men and women over a median of 23 years reported that increased BMI in adolescence was associated with increased risk of colorectal and rectal cancer (7). The mechanisms by which obesity increases cancer risk remain to be fully elucidated. However, research indicates that they are multifactorial, with sedentary lifestyle emerging as a major contributor (8). Two established factors driving cancer risk are immune system dysregulation and chronic low-grade inflammation; both of which are well described in obesity. Dysregulated inflammation leads to altered cytokine and adipokine profiles, which are intrinsically linked to cancer development $(9$, 10). Recently, we reported immune system dysregulation and chronic low-grade inflammation in a cohort of obese children with a mean age of 13 years (11). NK cells are innate effector cells that are capable of carrying out potent cytotoxic actions against tumor cells without previous exposure (12). In addition to transformed 
Table 1. Cohort characteristics

\begin{tabular}{cccc}
\hline & Lean & Obese & P value \\
\hline$n$ & 45 & 45 & - \\
Age (yr [range]) & $12.42(6.5-16.52)$ & $13.12(6.14-16.83)$ & 0.58 \\
Sex (\% male) & $55.6 \%$ & $57.7 \%$ & - \\
\hline BMI Z score & $0.06(-2.3-1.5)$ & $2.82(1.07-4.5)$ & $<0.0001$ \\
Weight (kg) & $42.1(18.9-82)$ & $87.89(30-154)$ & $<0.0001$ \\
Height (cm) & $147.5(111-196)$ & $160.5(124-182)$ & $<0.01$ \\
\hline HOMA-IR & $0.74(0.28-4.4)$ & $3.29(0.33-16.1)$ & $<0.0001$ \\
\hline Fasting glucose (mmol/l) & $5.02(3.5-5.6)$ & $5.03(3.4-6.6)$ & 0.93 \\
Fasting insulin (mmol/l) & $25.2(13-81)$ & $139(11-900)$ & $<0.0001$ \\
\hline
\end{tabular}

tumor cells, NK cells can respond to allogeneic, virus-infected, and stressed cells $(13,14)$. NK cells are early potent producers of IFN- $\gamma$, a cytokine that plays a key role in tumor immunosurveillance and activates the inflammatory cascade against neoplastic threat (15). In patients and animal models with impaired or depleted NK cells, there is a strong association with recurring viral infections and incidence of certain cancers $(16,17)$. NK cells are defined by their expression of the surface molecule CD56 and the lack of the T cell marker CD3. NK cells can be further separated in CD56 bright and CD56 $6^{\mathrm{dim}}$ subsets. CD56 bright NK cells are considered to be less cytotoxic than their dim counterparts but do show increased cytokine production. Given that NK cell numbers are decreased and display functional impairment in obese adults, we hypothesized that these deficits may also be present in a cohort of obese children (18-20). Furthermore, analysis of pediatric patients allows examination of immune function prior to onset of metabolic complications such as T2DM and thus may provide a good model for identification of mechanisms of obesity-related impaired immune function. Finally, it has recently been demonstrated that NK cell functions are affected by intrinsic cell metabolism $(21,22)$. Therefore, we hypothesized that the altered environment in childhood obesity might affect NK cell metabolism and drive dysfunction.

\section{Results}

We initially investigated the frequencies of NK cells in a cohort of obese and lean children aged 6-17 years of age (clinical cohort described in Table 1). NK cell frequencies and absolute numbers were reduced in obese children (Figure 1, A-C). NK cell frequencies negatively correlated with BMI $Z$ score (Figure 1, D and E). Homeostatic model of assessment for insulin resistance (HOMA-IR) calculations demonstrated significantly higher levels of insulin resistance in our obese cohort, which coincided with a significant decrease in NK cell frequencies in children with a HOMA-IR of greater than 3.1, indicative of an insulin-resistant state (23) (Figure 1, F and G). No associations were observed between NK cell frequencies and total cholesterol (Supplemental Figure 1E; supplemental material available online with this article; https://doi.org/10.1172/jci. insight.94939DS1). Additionally, pubertal status did not affect the frequencies of NK cells (data not shown). Obese children displayed an increase in CD56 $6^{\text {bright }}$ (cytokine-producing) NK cells, with a corresponding reduction in the frequency of CD56 $6^{\mathrm{dim}}$ (cytotoxic) NK cells (Supplemental Figure 1, B-D).

In addition to NK cell frequencies, we investigated a panel of NK cell-activating and -inhibitory molecules. Of the molecules investigated, only CD69 and program death-1 (PD-1) displayed differences (Figure 2 and Supplemental Figure 2). NK cells from obese children expressed higher basal levels of the activation marker CD69 (Figure 2A). Obese children also expressed higher levels of the exhaustion marker PD-1 following cytokine stimulation on their NK cells (Figure 2, B-D). To investigate if obesity in the absence of comorbidities and/or polypharmacy affected NK cell effector functions, we isolated NK cells from both lean and obese children and challenged their functional abilities in vitro following stimulation with two key cytokines (IL-15 and IL2) necessary for cellular proliferation and survival. NK cells isolated from lean children displayed consistent proliferation responses to these cytokines, with a significant increase in cell number on day 7 (Figure 2, E and G). In contrast, NK cells isolated from obese children did not proliferate consistently and failed to significantly expand after 7 days (Figure 2, F and G).

The primary effector function of NK cells is their ability to lyse transformed or virally infected cells. We investigated this function by coculturing NK cells with a NK cell-sensitive tumor cell line, K562, 
A

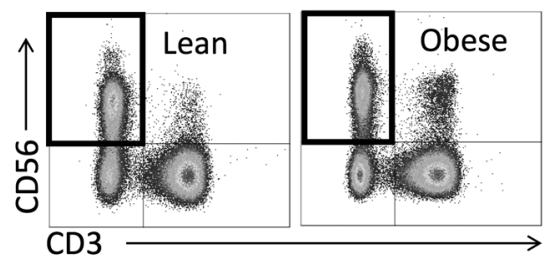

B

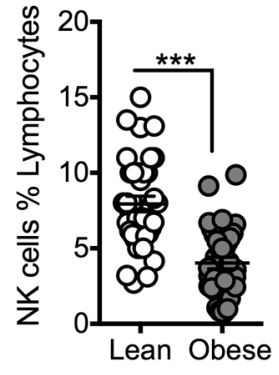

C

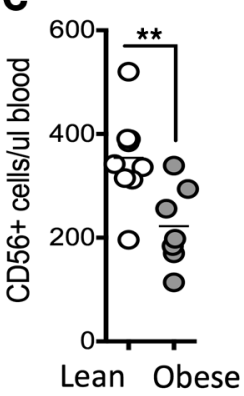

D

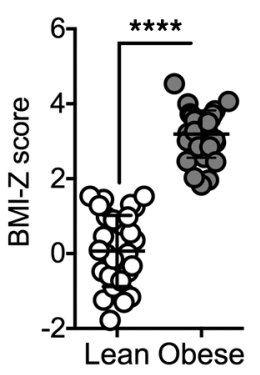

E

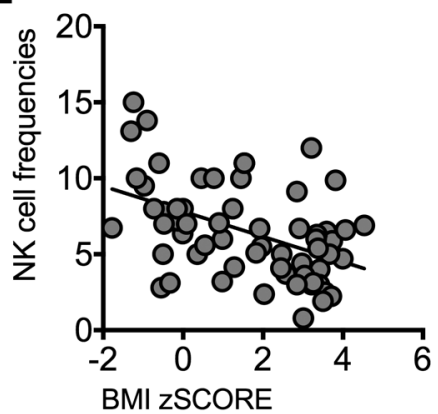

Figure 1. NK cell frequencies are reduced in childhood obesity. (A) Representative dot plots showing NK cells from a lean and an obese child. (B) Scatter plot showing NK cell frequencies (as a percentage of total lymphocytes) in lean ( $n$ $=35)$ and obese $(n=35)$ childhood cohorts. (c) Scatter plot displaying absolute number of NK cells ( $C D 56^{+}$cells $/ \mu$ l of blood) in a cohort of lean and obese children ( $n=10 /$ cohort). (D) Scatter plot detailing the BMI Z score of the lean and obese cohorts, and $(\mathbf{E})$ correlation plot showing the negative association between NK cell frequencies and BMI $Z$ score (Pearson $\mathrm{R}=-0.465, P=0.0002$ ). ( $F$ ) Scatter plot detailing the HOMA-IR score of the lean and obese childhood cohorts. (C) Scatter plots detailing the frequencies of NK cells in obese children separated according to HOMA-IR insulin-sensitive $(<3.1)$ and insulin-resistant $(>3.1)$ groups. Statistical comparisons using Student's $t$ test. ${ }^{* *} P \leq 0.01$, ${ }^{* *} P<$ $0.001,{ }^{* * *} P<0.0001$.
F

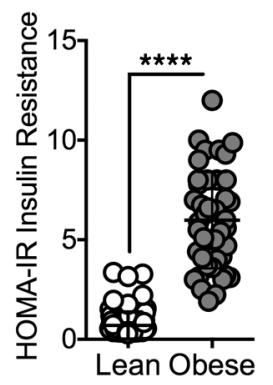

G

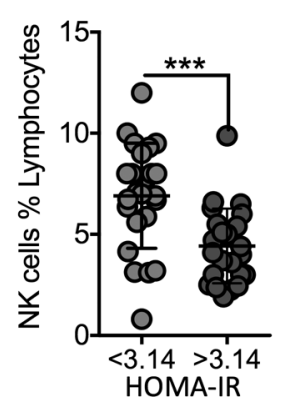

for 4 hours. NK cell numbers were normalized to account for the reduction in cell numbers in obese children. NK cells isolated from obese children killed significantly fewer K562 tumor cells compared with their lean counterparts (Figure $2 \mathrm{H}$ ). We also investigated lytic molecule expression by NK cells after coculture with K562 and showed that NK cells from obese children express reduced levels of both granzyme B and perforin when compared with lean controls (Figure 2, I-L).

NK cells can also exert their effector function via the production of cytokines, in particular IFN- $\gamma$. Therefore, we next examined the capacity of both lean and obese NK cells to produce IFN- $\gamma$ following cytokine stimulation. When challenged, NK cells isolated from both lean and obese children readily produced IFN- $\gamma$ (Figure 2, M and N).

Recent studies have shown that NK cell effector function is intrinsically linked to cell metabolism (21, 22). NK cells rely on metabolic reprogramming for effector function. Inhibition of either glycolysis or the glycolytic regulator mammalian target of rapamycin complex 1 (mTORC1) results in diminished effector responses by NK cells, such as cytokine production $(21,22)$. Therefore, we investigated the effect of obesity on pediatric NK cell metabolic reprogramming. Initially, we examined the expression of a major glucose transport, Glut-1, on NK cells and found no difference between lean and obese cohorts (Figure 3A). We next assessed the ability of NK cells to take up a fluorescent glucose analog, 2-NBDG. We observed increased 2-NBDG uptake by NK cells isolated from lean children upon activation but noted that NK cells from obese children had a trend toward increased basal glucose consumption (Figure 3, B and C).

As NK cells use mTOR for nutrient sensing and metabolic reprogramming, we next studied the activation of mTORC1 via the phosphorylation of ribosomal S6 protein (pS6). NK cells from obese children displayed higher levels of mTORC1 activation both basally and after 4 hours of cytokine stimulation, when compared with NK cells from lean children. Following 18 hours of stimulation, obese NK cell 
A

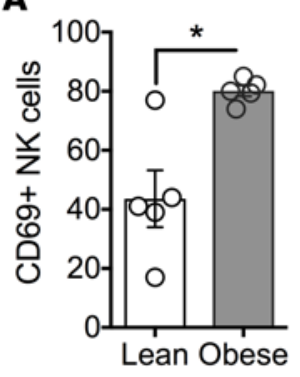

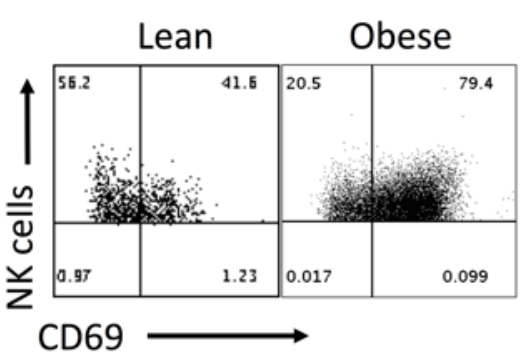

E

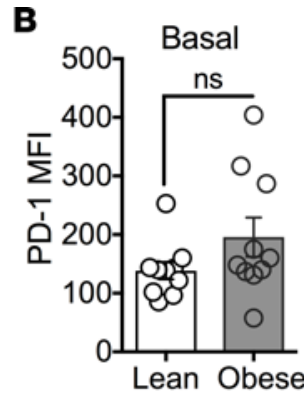

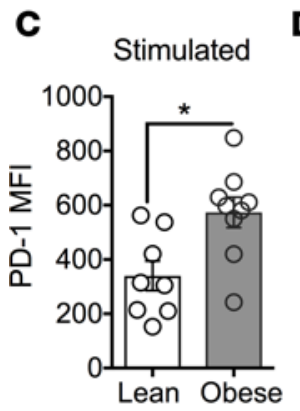

D
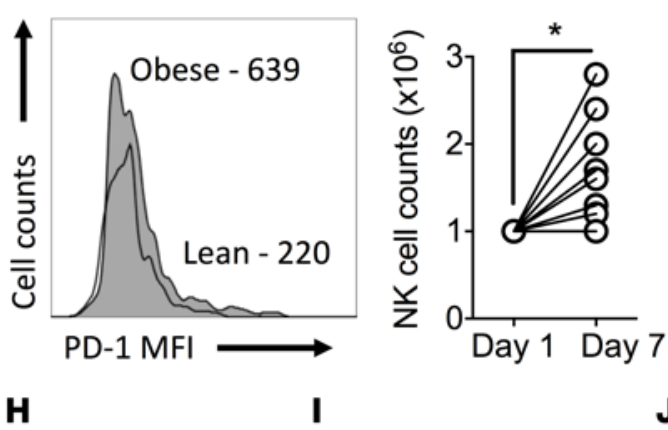

$\mathbf{F}$

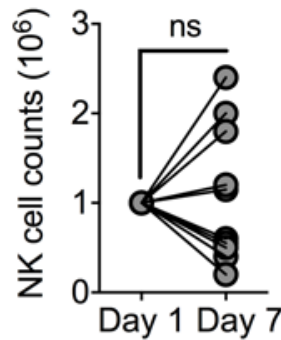

G
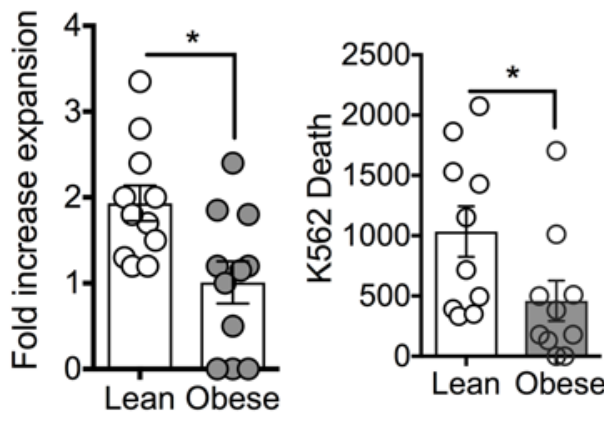

J

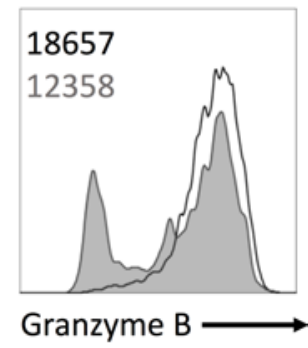

$\mathbf{L}$

M
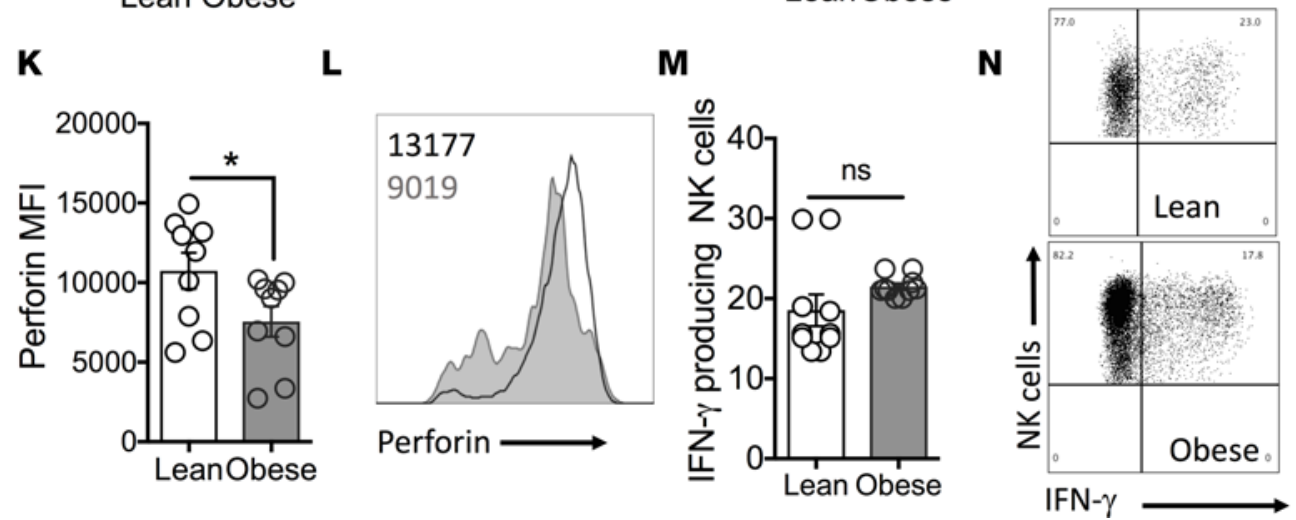

Figure 2. NK cells from obese children display increased activation and diminished effector responses. (A) Bar graph and representative dot detailing the percentage of NK cells expressing CD69 in lean and obese childhood cohorts $(n=5)$. Bar graphs showing (B) basal or (C) IL-2/IL-12-stimulated PD-1 expression (MFI) on NK cells from lean and obese children. (D) Representative dot plot showing PD-1 expression on stimulated NK cells from a lean and an obese donor. The numbers represent the MFI for the histograms on which they are displayed, the black corresponds to leans as per the histogram and grey represents obese MFI (D, J, and L). Plots showing the expansion of NK cells from (E) lean and (F) obese children following 7 days of IL-2/IL-15 stimulation. (C) Scatter plot showing the fold expansion (over baseline numbers) of NK cells from lean and obese children stimulated with IL-2/IL-15 for 7 days. (H) Bar graph showing the number of K562 tumor cells lysed (10:1 effector/target ratio) in 4 hours by NK cells isolated from either lean or obese children. (I and J) Bar graph and representative histogram showing granzyme B levels on K562 cells cocultured with NK cells from lean or obese children. (K and $\mathbf{L}$ ) Bar graph and representative histogram showing perforin levels on K562 stimulated NK cells from lean or obese children. (M) Bar graph showing IL-2/IL-12stimulated NK cell production of IFN- $\gamma$ in a cohort of lean and obese children. (N) Representative dot plot showing IFN- $\gamma$ production by NK cells basally or stimulated with IL-2/IL-12 for 18 hours. Data are representative of a minimum of 10 independent experiments. Statistical comparisons using Student's $t$ test. ${ }^{*} P<0.05,{ }^{* *} P \leq 0.01$. 

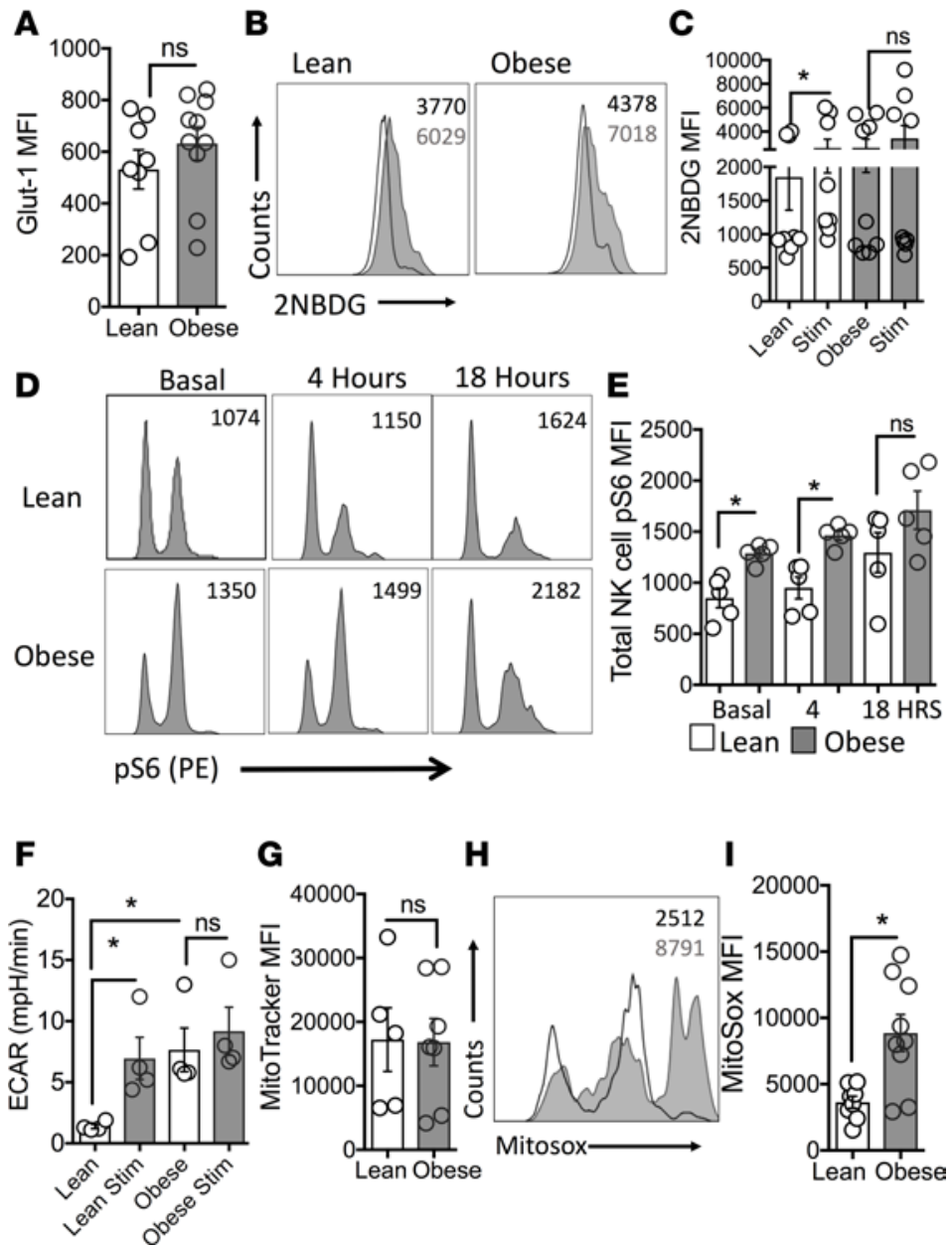

Figure 3. NK cells from obese children display increased mTORC1 activity, ECAR, and mitochondrial ROS. (A) Bar graph detailing the expression (MFI) of Glut-1 by NK cells from lean and obese children ( $n=10$ obese). (B and C) Representative histogram and bar graphs showing basal or stimulated 2-NBDC uptake by NK cells isolated from lean and obese children. The numbers represent the MFI for the histograms on which they are displayed, the black corresponds to leans as per the histogram and grey represents obese MFI. (D) Representative histograms and (E) bar graphs detailing MFI of phosphorylation of ribosomal S6 protein (pS6) expression (mTORC1 activity) in NK cells from lean or obese children. Expression of pS6 was measured in resting NK cells (basal) and cytokine-stimulated NK cells after 4 hours or 18 hours $(n=5)$. (F) Bar graph showing the extracellular acidification rate (ECAR) of basal or stimulated NK cells from lean or obese children $(n=4)$. (G) Bar graph showing the mitochondrial mass (MitoTracker Green) of NK cells from lean and obese children. (H) Representative histogram showing mitochondrial ROS (MitoSOX) MFI in NK cells from a lean and an obese child ( $n=9$ obese). The numbers represent MFI for the histograms. The black font represents lean NK cell mitosox MFI value and grey is the obese. (I) Bar graph showing mitochondrial ROS levels (MFI) in NK cells from lean and obese children. Data are representative of a minimum of 10 independent experiments, unless otherwise noted. Statistical comparisons using Student's $t$ test or ANOVA with multiple comparisons for groups of 3 or more data sets. ${ }^{*} P<0.05$.

mTORC1 activity did not significantly increase over basal levels (Figure 3, D and E, and Supplemental Figure 3). We next utilized Seahorse XF technology to investigate NK cell glycolysis rates in a subset of lean and obese children. Similar to the mTORC1 profile, NK cells isolated from obese children displayed elevated basal extracellular acidification rates (ECARs; a surrogate measure of glycolysis) levels compared with NK cells from lean children (Figure 3F).

Previous studies have shown that, upon activation, murine NK cells display increased mitochondrial stress in the form of increased ROS, followed by contraction in mitochondrial mass, due to increased mitophagy (24). We investigated if the apparent chronic metabolic activation of NK cells isolated from obese children affected mitochondrial frequencies or stress. We found that NK cells isolated from obese children showed no difference in mitochondrial mass compared with lean children, as measured by MitoTracker (Figure 3G), but did show increased mitochondrial ROS, measured by MitoSOX (Figure 3, H and I).

\section{Discussion}

In this study, we show that in obese children NK cells are reduced in frequency, display an activated metabolic phenotype, and are losing their ability to exert their effector functions, such as cytotoxicity. NK cells are a critical component of the immune system. Their primary functions are in antitumor and antiviral defenses. NK cells have an innate ability to recognize infected or transformed cells and subsequently kill the target cell, while initiating an adaptive immune response if required. We show that NK cells are reduced in the peripheral blood of obese children when compared with a cohort of lean counterparts. Previously, we reported a reduction in NK cell frequencies in a cohort of severely obese adults. In the same study, we showed that NK cell defects were more pronounced in metabolically unhealthy obese adults than metabolically healthy obese adults (25). This fits with the findings of the current study that the reduction in NK cell frequencies correlated negatively with BMI $Z$ score and insulin resistance. We show that childhood obesity negatively affects cytokine-driven NK cell proliferation and suggest that this may explain the reduced frequencies of NK cells observed in our obese cohort.

NK cells from obese children expressed higher levels of the activation marker CD69 and, upon stimulation, significantly upregulated PD-1, indicating that these cells are highly activated and become exhausted much quicker when challenged, compared with their lean counterparts. Guo and colleagues reported that NK cells expanded in the presence of a blocking antibody against PD-1, demonstrating increased cytolytic activ- 
ity against myeloma cells (26). We also examined the NK cell-related molecules NKG2D, TIM-3, CD158a, CD158b, CD16, CD25, and CD95 and identified no difference between lean and obese children. A recent study by Theurich and colleagues reported no difference between lean and obese NK cells in their expression of many classical NK cell molecules, such as the NKG2 family (20).

Previous studies in obese adults with T2DM reported a loss of NK cell tumor lysis capabilities (18). Our childhood cohort allows us to investigate the effect of obesity on the immune system, outside of comorbidities and polypharmacy. We show that NK cells isolated from obese children display dysregulated expansion capabilities; a subset of our obese cohort displayed normal expansion, while NK cells of several patients in the cohort displayed a complete failure to expand. We show that NK cells isolated from obese children display reduced tumor lysis capabilities and lytic molecule production. A prospective study has demonstrated a relationship between the natural cytotoxicity of peripheral blood mononuclear cells (PBMCs) and cancer risk, identifying those with the lowest cytotoxic activity as having the highest cancer risk. This finding suggests that the loss of NK cell cytotoxicity in obesity may contribute to the future risk of developing disease (16). Due to the small sample volume available from children, we did not investigate antibody-dependent cellular cytotoxicity, which remains to be elucidated in childhood obesity. Encouragingly, a study investigating NK cell cytotoxic function before and after bariatric surgery found that defective NK cell cytotoxicity observed in a cohort of severely obese adults improved after surgery (27). This suggests the defects in NK cell cytotoxic function that are driven by obesity are reversible.

Upon activation, NK cells undergo metabolic reprogramming, increasing rates of both oxidative phosphorylation and aerobic glycolysis (28). This reprogramming ensures that NK cells have the energy and biological intermediates required to produce effector molecules, such as lytic granules and cytokines. We demonstrated that NK cells isolated from obese children have altered cellular metabolism when compared with lean counterparts. We observed increased 2-NBDG uptake by NK cells isolated from lean children upon activation but did not see this in NK cells from obese children. However, we noted that NK cells from obese children had a trend toward increased basal 2-NBDG uptake, in agreement with the concept of an activated population. NK cells utilize mTOR for metabolic reprogramming. We discovered that the activation of mTORC1 was higher in NK cells from obese children both basally and after 4 hours of stimulation, supporting our findings of an activated phenotype. Following 18 hours of stimulation, obese NK cell mTORC1 activity did not significantly increase, unlike that in lean counterparts, suggesting that NK cells from obese children are maximally activated in the basal state. Similar to these findings, in a model of LCMV infection, the Wherry group reported elevated mTORC1 activity in T cells from mice with chronic LCMV infection and a subsequent reduction in responsiveness to stimulation (29). To confirm the concept of a metabolically activated NK cell, we performed Seahorse analysis on a subset of our cohort. We showed that basal ECARs, a surrogate for glycolysis, are elevated in NK cells from obese children and were not increased upon stimulation, consistent with our mTORC1 findings.

Keating and colleagues have recently shown that NK cell function is also highly dependent on mitochondrial OxPhos (28). We demonstrated large increases in mitochondrial ROS in NK cells from obese children, indicating mitochondrial stress, suggesting that possible dysfunction may contribute to alterations in these cells. Data from the Sun lab has shown that upon activation murine NK cells display increased mitochondrial stress in the form of increased ROS (24). These data also show that survival of these murine NK cells relied upon mitophagy-mediated clearance of these dysfunctional mitochondria (24). Therefore, the observed mitochondrial dysfunction in NK cells from obese children may contribute to the observed decreased NK cell numbers and impaired effector function. Indeed, several studies in T lymphocytes, in the context of chronic viral infection, support the argument that mitochondrial dysfunction is linked to lymphocyte dysregulation (29-31). Fisicaro and colleagues demonstrated that chronic hepatitis B infection resulted in exhausted dysfunctional CD8 T cells, which expressed PD-1 and displayed mitochondrial defects, including elevated mitochondrial ROS, drawing striking similarities to the effect of obesity on NK cells (30).

Overall, our data show that obese children have a reduced population of circulating NK cells that are highly activated, metabolically stressed, and underperforming in their basic duties. These observations prove that the negative effect of obesity on antitumor immunity is present in children.

\section{Methods}

Study cohorts. A cohort of 90 children (45 obese/ 45 nonobese) aged between 6 and 17 years were recruited (Table 1). Experiments detailed in Figures 2 and 3 were performed on a subset of these cohorts (numbers as denoted in figure legends). Clinical assessments (height, weight, fasting insulin, fasting glucose, lipid 
profiles) were performed as part of the participants' routine care. Biochemical measurements (insulin, glucose, lipids, and absolute cell numbers) were measured by the clinical Biochemistry and Haematology labs at Our Lady's Children's Hospital Crumlin. Inclusion and exclusion criteria and the clinical investigation protocol are as previously described (11).

Preparation of PBMCS and flow cytometric analysis. PBMC samples were isolated by density centrifugation. NK cell staining $\left(1 \times 10^{6} \mathrm{PBMCs}\right)$ was performed using specific surface monoclonal antibodies, namely CD56 PerCp-Vio700 (Miltenyi Biotec, clone REA196) and CD3 VioGreen (Miltenyi Biotec, clone REA613), and represented as percentages of total lymphocytes (unless otherwise stated). NK cells were defined as $\mathrm{CD}^{2} 6^{+}, \mathrm{CD}^{-}$lymphocytes. Other markers of $\mathrm{NK}$ cell activation and exhaustion examined were CD69 APC-Vio770 (Miltenyi Biotec, clone FN50), PD-1 PE-Vio770 (Miltenyi Biotec, clone PD1.3.1.3), CD25 APC (BD Biosciences, clone M-A251), CD16 PE-Vio770 (Miltenyi Biotec, clone REA423), NKG2D APC (BD Biosciences, clone 1D11), CD158a PE (BD Biosciences, clone HP-3E4), and CD158b FITC (BD Biosciences, clone $\mathrm{CH}-\mathrm{L}$ ). Cell populations were acquired using a BD FACSCanto II and analyzed using FlowJo software (Treestar). Results are expressed as a percentage of the parent population as indicated and determined using flow minus-1 (FMO) and unstained controls.

NK cell proliferation analysis. NK cells were isolated from donor PBMC populations using a positive selection MACS Magnetic Bead (Miltenyi Biotec) isolation technique, as per the manufacturer's instructions. NK cells were cultured $\left(1 \times 10^{6}\right.$ cells $\left./ \mathrm{ml}\right)$ in the presence of IL-15 $(5 \mathrm{ng} / \mathrm{ml})$ and IL-2 $(20 \mathrm{ng} / \mathrm{ml})$ for 7 days. NK cell numbers were determined using the Invitrogen Countess system.

NK cell cytotoxicity analysis. PBMCs (for granzyme B and perforin) or isolated NK cells (for tumor lysis) were cultured with K562 cell line (ATCC), a myelogenous leukemia cell line, at a ratio of 10:1, respectively, for 4 hours at $37^{\circ} \mathrm{C}$. Granzyme B (APC, Miltenyi Biotec, clone REA226) and perforin (VioBlue, Miltenyi Biotec, clone $\delta$ G9) expression was determined by flow cytometry. NK cell cytotoxicity was determined using the CytoTox-Fluor cytotoxicity assay (Promega), as per the manufacturer's instructions.

NK cell cytokine production analysis. Isolated NK cells $\left(1 \times 10^{6}\right.$ cells $\left./ \mathrm{ml}\right)$ were stimulated with IL-15 (5 $\mathrm{ng} / \mathrm{ml})$, IL-2 $(20 \mathrm{ng} / \mathrm{ml})$, and IL-12 $(30 \mathrm{ng} / \mathrm{ml})$ for 18 hours at $37^{\circ} \mathrm{C}$. Culture supernatants were investigated for IFN- $\gamma$ secretion by ELISA (Biolegend), as per the manufacturer's instructions.

NK cell glucose consumption analysis. PBMCs or isolated NK cells $\left(1 \times 10^{6}\right.$ cells $\left./ \mathrm{ml}\right)$ were activated as previously described for 18 hours. Cells were washed and transferred to glucose-restricted media in the presence of the fluorescent glucose analog 2-NBDG (Life Technologies) for 2 hours. Additionally, Glut-1 expression was examined on activated PBMCs or isolated NK cells using specific fluorescently labeled $\mathrm{mAb}$ (R\&D Systems). Glucose uptake and Glut-1 expression were determined using flow cytometry. FMO and unstimulated NK cells were used as negative controls.

NK cell mTORC1 and mitochondrial analysis. PBMCs $\left(1 \times 10^{6}\right.$ cells $\left./ \mathrm{ml}\right)$ were activated as previously described for 4 or 18 hours. mTORC1 activity in NK cells $\left(\mathrm{CD}^{+} 6^{+}, \mathrm{CD}^{-}\right)$was examined via pS6 (Cell Signalling) by flow cytometry. Additionally, NK cell mitochondrial mass (MitoTracker) and stress (MitoSOX) were examined. Briefly, prior to NK cell surface staining on PBMCs, cells were stained with MitoTracker Green FM (200 nM) or MitoSOX Red $(5 \mu \mathrm{M})$ for 30 minutes at $37^{\circ} \mathrm{C}$, as per the manufacturer's instructions. Fully stained cells were analyzed by flow cytometry. FMO and unstimulated NK cells were used as negative controls.

NK cell Seahorse analysis. Isolated NK cells were activated as previously described for 18 hours. After cell stimulation, the ECAR was measured in 250,000 purified NK cells per well using a Glycolysis Stress Test Kit and an XFp Extracellular Flux Analyzer, according to the manufacturer's instructions (Seahorse XF96 Technology, Agilent). Each cell culture condition was evaluated in quadruplicate, and 12 measures were made per sample. The ECAR linked with basal glycolysis pathway activation was measured after the addition of glucose $(10 \mathrm{mM})$ in cell culture.

Statistics. Statistical analysis was completed using GraphPad Prism 6 software. Data are expressed as SEM. We determined differences between two groups using 2-tailed Student's $t$ test and Mann-Whitney $U$ test where appropriate. Differences between three or more groups were determined using ANOVA with multiple comparisons. Correlations were determined using linear regression models and expressed using Pearson or Spearman's rank correlation coefficient as appropriate. $P$ values were expressed with significance set at $<0.05$.

Study approval. Ethical approval was granted by the Medical Research Ethics Committees at Our Lady's Children's Hospital Crumlin. The parents of all pediatric patients gave written informed consent prior to patient participation in the study. 


\section{Author contributions}

LMT, MM, EC, and DK performed the experiments, carried out analysis, and approved the final manuscript as submitted. ECO and MAL performed and analyzed the Seahorse experiments. AEH, DKF, DC, and DOS conceptualized and designed the study, analyzed the data, drafted the manuscript, and approved the final manuscript as submitted.

\section{Acknowledgments}

This study is supported by a grant from the National Children's Research Centre, Crumlin, Ireland. Additionally, this study was supported in part by HRB Centre for Health \& Diet Research (grant HRC2014/13) and a Health Research Award (grant RA-POR-2014-593), both of which are funded by the Irish Health Research Board.

Address correspondence to: Andrew Hogan, Institute of Immunology, Department of Biology, Maynooth University, Biosciences Building, Room B2.16, Maynooth, Co Kildare, Ireland. Phone: 01.7086118; Email: Andrew.E.Hogan@mu.ie.

1. Ferraro KF, Thorpe RJ, Wilkinson JA. The life course of severe obesity: does childhood overweight matter? J Gerontol B Psychol Sci Soc Sci. 2003;58(2):S110-S119.

2. Calle EE, Rodriguez C, Walker-Thurmond K, Thun MJ. Overweight, obesity, and mortality from cancer in a prospectively studied cohort of U.S. adults. NEngl J Med. 2003;348(17):1625-1638.

3. Renehan AG, Tyson M, Egger M, Heller RF, Zwahlen M. Body-mass index and incidence of cancer: a systematic review and meta-analysis of prospective observational studies. Lancet. 2008;371(9612):569-578.

4. Butturini AM, et al. Obesity and outcome in pediatric acute lymphoblastic leukemia. J Clin Oncol. 2007;25(15):2063-2069.

5. Gelelete CB, et al. Overweight as a prognostic factor in children with acute lymphoblastic leukemia. Obesity (Silver Spring). 2011;19(9):1908-1911.

6. Bertrand KA, Giovannucci E, Zhang SM, Laden F, Rosner B, Birmann BM. A prospective analysis of body size during childhood, adolescence, and adulthood and risk of non-Hodgkin lymphoma. Cancer Prev Res (Phila). 2013;6(8):864-873.

7. Levi Z, et al. Adolescent body mass index risk of colon rectal cancer in a cohort of 1.79 million Israeli men and women: A population-based study. Cancer. 2017;123(20):4022-4030.

8. Taubes G. Cancer research. Unraveling the obesity-cancer connection. Science. 2012;335(6064):28, 30-32.

9. Osborn O, Olefsky JM. The cellular and signaling networks linking the immune system and metabolism in disease. Nat Med. 2012;18(3):363-374.

10. van Kruijsdijk RC, van der Wall E, Visseren FL. Obesity and cancer: the role of dysfunctional adipose tissue. Cancer Epidemiol Biomarkers Prev. 2009;18(10):2569-2578.

11. Carolan E, et al. The impact of childhood obesity on inflammation, innate immune cell frequency, and metabolic microRNA expression. J Clin Endocrinol Metab. 2014;99(3):E474-E478.

12. Swann JB, Smyth MJ. Immune surveillance of tumors. J Clin Invest. 2007;117(5):1137-1146.

13. Herberman RB, Nunn ME, Holden HT, Lavrin DH. Natural cytotoxic reactivity of mouse lymphoid cells against syngeneic and allogeneic tumors. II. Characterization of effector cells. Int J Cancer. 1975;16(2):230-239.

14. Wensveen FM, et al. NK cells link obesity-induced adipose stress to inflammation and insulin resistance. Nat Immunol. 2015;16(4):376-385.

15. Dunn GP, Koebel CM, Schreiber RD. Interferons, immunity and cancer immunoediting. Nat Rev Immunol. 2006;6(11):836-848.

16. Imai K, Matsuyama S, Miyake S, Suga K, Nakachi K. Natural cytotoxic activity of peripheral-blood lymphocytes and cancer incidence: an 11-year follow-up study of a general population. Lancet. 2000;356(9244):1795-1799.

17. Orange JS. Natural killer cell deficiency. J Allergy Clin Immunol. 2013;132(3):515-525; quiz 526.

18. O'Shea D, Cawood TJ, O'Farrelly C, Lynch L. Natural killer cells in obesity: impaired function and increased susceptibility to the effects of cigarette smoke. PLoS ONE. 2010;5(1):e8660.

19. O'Rourke RW, Gaston GD, Meyer KA, White AE, Marks DL. Adipose tissue NK cells manifest an activated phenotype in human obesity. Metab Clin Exp. 2013;62(11):1557-1561.

20. Theurich S, et al. IL-6/Stat3-dependent induction of a distinct, obesity-associated NK cell subpopulation deteriorates energy and glucose homeostasis. Cell Metab. 2017;26(1):171-184.e6.

21. Donnelly RP, et al. mTORC1-dependent metabolic reprogramming is a prerequisite for NK cell effector function. J Immunol. 2014;193(9):4477-4484.

22. Marçais A, Walzer T. mTOR: a gate to NK cell maturation and activation. Cell Cycle. 2014;13(21):3315-3316.

23. Keskin M, Kurtoglu S, Kendirci M, Atabek ME, Yazici C. Homeostasis model assessment is more reliable than the fasting glucose/insulin ratio and quantitative insulin sensitivity check index for assessing insulin resistance among obese children and adolescents. Pediatrics. 2005;115(4):e500-e503.

24. O'Sullivan TE, Johnson LR, Kang HH, Sun JC. BNIP3- and BNIP3L-mediated mitophagy promotes the generation of natural killer cell memory. Immunity. 2015;43(2):331-342.

25. Lynch LA, O'Connell JM, Kwasnik AK, Cawood TJ, O'Farrelly C, O'Shea DB. Are natural killer cells protecting the metabolically healthy obese patient? Obesity (Silver Spring). 2009;17(3):601-605.

26. Guo Y, et al. PD1 blockade enhances cytotoxicity of in vitro expanded natural killer cells towards myeloma cells. Oncotarget 2016;7(30):48360-48374.

27. Moulin CM, Marguti I, Peron JP, Halpern A, Rizzo LV. Bariatric surgery reverses natural killer (NK) cell activity and NK-relat- 
ed cytokine synthesis impairment induced by morbid obesity. Obes Surg. 2011;21(1):112-118.

28. Keating SE, et al. Metabolic reprogramming supports IFN- $\gamma$ production by CD56bright NK cells. J Immunol. 2016;196(6):2552-2560.

29. Bengsch B, et al. Bioenergetic insufficiencies due to metabolic alterations regulated by the inhibitory receptor PD-1 are an early driver of CD8(+) T cell exhaustion. Immunity. 2016;45(2):358-373.

30. Fisicaro $\mathrm{P}$, et al. Targeting mitochondrial dysfunction can restore antiviral activity of exhausted HBV-specific CD8 $\mathrm{T}$ cells in chronic hepatitis B. Nat Med. 2017;23(3):327-336.

31. Schurich A, et al. Distinct metabolic requirements of exhausted and functional virus-specific CD8 T cells in the same host. Cell Rep. 2016;16(5):1243-1252. 